\title{
GPPS-CH-2020-0155
}

\section{RESEARCH ON THE EFFECT OF PRESSURE SIDE WINGLET PROFILE ON THE PERFORMANCE OF HIGH-LOADED COMPRESSOR ROTOR}

\author{
Han TENG \\ Marine Engineering College \\ tenghangt@sina.com \\ Dalian, Liaoning, China
}

\author{
Jingjun ZHONG \\ Merchant Marine College \\ zhongjj@shmtu.edu.cn \\ Pudong, Shanghai, China
}

\author{
Wanyang WU \\ Merchant Marine College \\ wywu@shmtu.edu.cn \\ Pudong, Shanghai, China
}

\begin{abstract}
To improve the performance of an electric assistant turbocharger in different backpressure environments, the compressor margin needed to be optimized individually. As an effective passive control method to improve the margin, the full-chord-covered pressure side tip winglet has been studied and applied for compressor and turbine rotors. The research on the effect of partial tip winglets, which can reduce added mass of winglet and the weight of the rotor, on the compressor margin has not been found in the available literature. Utilizing numerical simulation, this paper was focused on the research of a high-speed single-rotor compressor which the tip loading coefficient is 0.81 . The shape of the pressure side tip winglet is optimized with the margin as the single objective. A database with 189 valid samples was generated using the Latin hypercube method. A self-developed $\varepsilon$-Support vector regression (SVR)-genetic algorithm (GA) optimization platform was applied to optimize the tip shape. The platform obtained a partial pressure side tip winglet shape. Compared with the CFD simulation results, the error of the $\varepsilon$-SVR-GA platform predicted the margin was $4.1 \%$. The result obtained by the $\varepsilon$-SVR-GA platform was improved compared with the prototype margin by $25.36 \%$.
\end{abstract}

\section{INTRODUCTION}

For the electrification process of vehicle power, regardless of electric vehicle battery technology or fuel cell technology, it is still difficult to compare it to the internal combustion engine in terms of cost control, difficulties with miniaturization, and technical reserve in a short time. Therefore, the development of energy-saving and low emission internal combustion engines to increase the proportion of electrification in the powertrain is still one of the key tasks for automotive power (HU et al. 2019). A serial electric auxiliary turbocharger (EAT) can be a reliable solution for turbo lag, which is caused by the non-instantaneous change of exhaust gas volume and temperature in the starting and transient stages, is mass-produced and equipped with an automotive engine, which is also favored by turbocharger manufacturers, OEM manufacturers and research institutions(Arnold et al. 2001; Katrašnik et al. 2003; Biwersi et al. 2012). Different from the exhaust gas-driven turbocharger, the EAT, which connects directly to the motor, is much more sensitive to the efficiency of the compressor rotor, when this increases, the driven motor output decreases. ZHAO et al. (2012) founded the improvement of the compressor rotor leads to the reduction of the output power demand of the motor. LIU et al. (2009) noticed that the rotational inertia of the compressor rotor limits acceleration ability and the time taken for the rotor to accelerate to the design speed also limits the performance of the EAT regarding the reduction of engine emissions and improvement of vehicle maneuverability. Due to the above problems, the research team proposed the idea of using low pressure ratio, low rotational inertia, and a high efficiency axial flow rotor for the design of an electrically assisted turbocharger rotor.

Since the inertia and compressibility of intake gas, the intake gas will produce both inertia and wave effects inside the manifold (SONG et al. 2015). This dynamic effect, which is reflected in the upstream of the EAT, is the compressor rotor outlet pressure periodical change. Due to the limitations of this working environment, the stability margin of the EAT rotor must be optimized. In vehicle engine rooms with limited space, active control methods such as plasma excitation and low energy fluid suction cannot be applied practically. A tip winglet, as a passive control method, can increase the stability margin of the compressor rotor significantly while slightly reducing pressure ratio and efficiency. Studies about compressor tip winglets mostly focus on the action mechanism of tip winglets in plane cascade (HAN et al. 2012; SANG 2017; WU et al. 2017), the different effects of suction side winglets and pressure side winglets (WU 2018), and the influence of varying locations and different maximum widths on the effect of winglet (ZHONG et al. 2016). The design criteria for compressor winglets has not been studied. Different compressor blade tips have different loads, it is 
difficult to establish a unified design method for compressor tip winglets. Even though it is easy to obtain a better form for engineering application through the use of the arrangement and combination of different winglets, widths and positions, because of the discreteness of combination, the best effect of winglets cannot be fully utilized. Therefore, the way to strike a balance between design time and the best result is a topic worth discussing. With current research, the shape of pressure surface winglets is a full chord length winglet. Whether such shaped winglets are necessary and whether partial winglets can achieve the same effect or better effect remains to be explored.

By summarizing earlier studies, this paper designed the shape of the pressure side winglet for the EAT rotor. In order to reduce the required amount of calculation, an $\varepsilon$-SVR support vector regression model was used as the agent model in order to calculate the objective function, and a genetic algorithm (GA) was used in order to optimize the penalty parameter $\mathrm{C}$, kernel function parameter $\mathrm{g}$ and the value $\mathrm{p}$ of the loss function in the $\varepsilon$-SVR model. The Latin hypercube experimental design method and a genetic algorithm were combined to optimize the design with stability margin as a single objective, and an $\varepsilon$-SVR-GA optimal design platform was constructed. The result demonstrated that the $\varepsilon-S V R-$ GA optimal design platform used in this paper can reduce the required sample size and improve the efficiency of optimal design. And the partial winglets can expand the stability margin with slight reduction in efficiency and pressure ratio. At the same time, partial winglets also add less additional mass into the rotor, which is an appropriate passive flow control method for the EAT rotor.

\section{METHODOLOGY}

\section{Optimization Object and Numerical Method}

The optimization object of this paper was a self-designed axial-flow EAT rotor. To increase the compressor load as much as possible, the flow channel with equal outside diameter was adopted. Table 1 shows the performance and geometric parameters of the prototype rotor at design point conditions. To improve the pressure ratio, with the condition of ensuring efficiency, the load of the hub, mean diameter and tip all exceeded the design limit of a conventional compressor (load coefficient $>0.6$ ).

Table 1 Geometric parameters and design point performance of EAT rotor

\begin{tabular}{llll}
\hline Parameters & Value & Parameters & Value \\
\hline External diameter $/ \mathrm{mm}$ & 83 & Mean diameter solidity & 1.64 \\
Inlet hub ratio & 0.45 & Mass flow /(Kg/s) & 0.629 \\
Rotation speed/rpm & 60000 & Pressure ratio & 1.346 \\
Mean diameter aspect ratio & 0.68 & Isentropic efficiency & $88.68 \%$ \\
\hline
\end{tabular}

In the numerical simulation, Numeca /Autogrid5 software was used for mesh generation, and Numeca /FINE was used in order to solve the steady Reynolds-Averaged Navier-Stokes (RANS) equation. The turbulence model was the Low-Re 1-equation model Spalart-Allmaras. The total inlet pressure was given as $101325 \mathrm{~Pa}$, the inlet direction was axial. The outlet conditions provided the static pressure at the mean radius position, and the static pressure at the other position was calculated based on the radial-equilibrium relation. The wall surface was set as an adiabatic non-slip wall surface, and a periodic boundary was used in order to calculate a single channel. The performance parameters of EAT in different states were calculated by changing the outlet back pressure. Following repeated adjustments, the calculation scheme was finally determined to increase the outlet backpressure by $3 \%$ in the stall direction and decrease the backpressure by $3 \%$ in the choke direction for each sample. A total of thirteen examples were used to obtain the stability margin of EAT. The mass flow stability margin is defined as

$$
\mathrm{SM}=\left(\frac{\pi_{\text {stall }} \times m_{\eta \max }}{\pi_{\eta \max } \times m_{\text {stall }}}-1\right) \times 100 \%
$$

In order to ensure the grid independence of the numerical solution, a total of four sets of grid numbers were drawn, the mesh numbers were $0.9 \times 10^{6}, 1.2 \times 10^{6}, 1.5 \times 10^{6}, 1.8 \times 10^{6}$, named by coarse, medium1, medium 2 and fine. After the calculation of the performance of the design points, the comparison demonstrated that when the number of grids reached $1.5 \times 10^{6}$, the performance parameters and flow field details were independent to the number of grids. This paper adopted a $1.5 \times 10^{6}$ grid for further research. Figure 1 demonstrates the tendency of performance parameters to vary with the number of grids. Figure 2 shows the prototype EAT grid. 


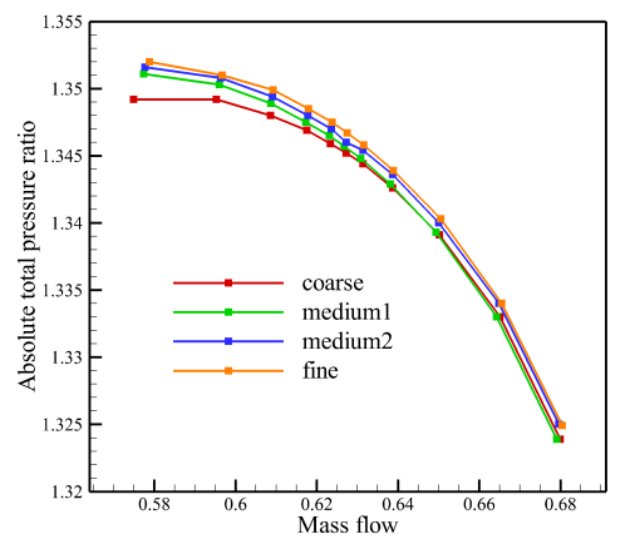

Figure 1. Grid independence research

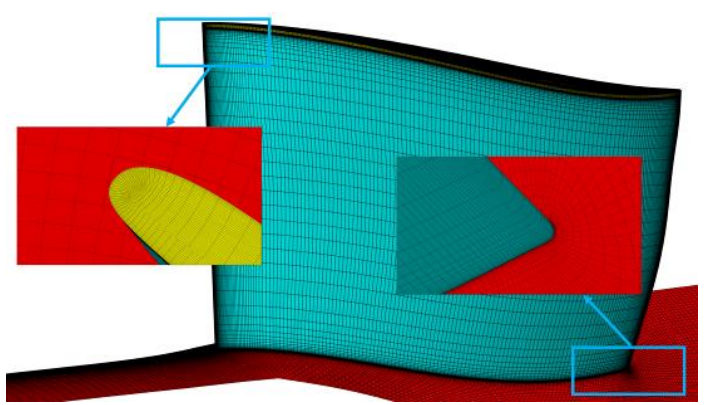

Figure 2. Prototype EAT grid

\section{Support Vector Regression}

Support vector machine (SVM) is a machine learning method which is based on statistical learning theory. It has a strong learning ability for nonlinear and small sample space problems and is used widely in pattern classification and function regression problems. When solving a function regression problem, SVM is also known as support vector regression (SVR). Its return value is not +1 or- 1 in the classification problem, but is continuous real numbers. With a regression problem, the following training sample is given:

We hope to obtain a regression model such as

$$
\mathrm{D}=\left\{\left(x_{1}, y_{1}\right),\left(x_{2}, y_{2}\right), \ldots \ldots\left(x_{m}, y_{m}\right)\right\}, y_{i} \in \mathbb{R}
$$

$$
f(x)=\omega^{T}+b
$$

, where $f(x)$ and $y$ are as close as possible, $\omega$ and $b$ are undetermined model parameters. As demonstrated in Figure 3 , the SVR assumes that we can tolerate the difference between $f(x)$ and $y$ when the absolute value of the loss is greater than $\varepsilon$. In other words, an interval band with a width of $2 \varepsilon$ was constructed as the center of $f(x)$. If the training sample fell within this interval band, it is then considered to have been correctly predicted.

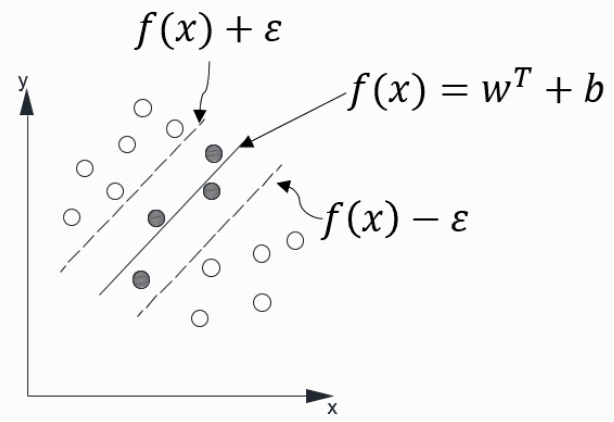

Figure 3. Schematic diagram of SVR

The SVR problem can be transformed into

$$
\min _{w, b} \frac{1}{2}\|w\|^{2}+C \sum_{i=1}^{m} \ell_{\varepsilon}\left(f\left(x_{i}\right)-y_{i}\right)
$$

where $\mathrm{C}$ is a regularization constant, $\ell_{\varepsilon}$ is $\varepsilon$ insensitive loss function.

$$
\ell_{\varepsilon}=\left\{\begin{array}{cc}
0, & \text { if }|z| \leq \varepsilon \\
|z|-\varepsilon, & \text { otherwise }
\end{array}\right.
$$

By introducing relaxation variables $\xi_{i}$ and $\widehat{\xi}_{l}$, equation (4) can be rewritten as

$$
\begin{aligned}
& \min _{w, b} \frac{1}{2}\|w\|^{2}+C \sum_{i=1}^{m}\left(\xi_{i}-\widehat{\xi}_{l}\right) \\
& y_{i}-\omega \cdot x_{i}-b \leq \varepsilon+\xi_{i} \\
& \text { S.T. } \omega \cdot x_{i}+b-y_{i} \leq \varepsilon+\widehat{\xi}_{l} \\
& \xi_{i}, \widehat{\xi}_{l} \geq 0 \quad i=1,2, \ldots, n
\end{aligned}
$$


an $\varepsilon$-SVR model is obtained. The original problem is an optimization problem with equality and inequality constraints. A Lagrange multiplier method is used to transform it into a dual problem, because the dual problem meets the KarushKuhn-Tucker (KKT) conditions, and the optimal solution of the original problem can be obtained by solving the dual problem. For nonlinear fitting, it is necessary to map the original sample to the high-dimensional space through nonlinear transformation, in order to the mapped sample to be fitted in the high-dimensional space. To avoid having to calculate the inner product in higher dimensions or even infinite dimensions, correct kernel functions must be constructed for the inner product of samples in higher dimensions to be equal to the result which is calculated using kernel functions in the original sample space. The most commonly used kernel functions are linear kernel, polynomial kernel, radial basis kernel (RBF) and sigmoid kernel. In this paper, an RBF kernel function is the preferred kernel function for the samples with six variables which corresponded to margin value change. Following the determination of the type of SVR model and kernel function, the parameters to be defined in the function setting are determined. In this case, the parameters to be selected included penalty parameter $\mathrm{C}$, kernel parameter $\mathrm{g}$ and the value $\mathrm{p}$ of the loss function in $\varepsilon-S \mathrm{R}$. In order to obtain the optimal parameters and maximize the use of valid samples, k-fold cross-validation was used. To evenly distribute the sample space, K was set as 9 .

\section{Pressure side winglet and rotor parameterization}

Winglets which can be used in the actual rotor are integrated winglets, that is to say that the winglets extend to the suction or pressure side and smooth transition with the blade. In a low-speed axial compressor, the pressure side winglet forces mainstream/tip leakage flow and mainstream interface to move towards the rotor's tailing edge. In one way, pressure side winglets reduce the leakage flow and active momentum ratio. in another way, it delays the suction side of the rotor blade boundary layer separation, reduces the blocking effect of low energy fluid on the blade channel, and expand the stability margin of the compressor rotor. In a transonic compressor, at speeds of $100 \%$ and $80 \%$, the pressure surface winglet can decrease the strength of shock wave/tip leakage vortex interference and reduce the blocking area caused by the tip leakage vortex breaking. At $60 \%$ design speed, the pressure side winglet can reduce the equivalent attack angle of the incoming flow and weaken the three-dimensional separation of the boundary layer on the suction surface. The design speed of EAT is $60,000 \mathrm{rpm}$, and because the hub ration is 0.45 , the tangential speed is high subsonic in the high spanwise location. To improve the stability margin of the EAT rotor, this paper carried out optimisation design of the pressure side winglet, whereas previous studies had all focused on the full-chord winglet. The main effect of the winglet is the inhibition of tip leakage flow, but according to the characteristics of tip leakage flow, strength is not constant along the chord direction. Whether the partial winglet which covers only part of the chord also inhibits the effect of leakage flow, and whether automatic optimisation will generate the partial winglet, are also worth exploring.

Before optimisation, prototype EAT first has to be parameterized. NUMECA/DESINGN3D software is used during the parameterization process, and the parameterized EAT model and prototype performance are calculated for comparison. Figure 4 shows the comparison between prototype EAT and parameterized EAT in mass flow-pressure ratio characteristic line and blade tip profile. This demonstrates that the performance of the parameterized EAT is the same close to the design point as that of the prototype EAT rotor. At the near stall point, the pressure ratio is slightly lower than that of the prototype EAT. The stability margin of prototype EAT is $0.07 \%$ less than parameterized EAT.

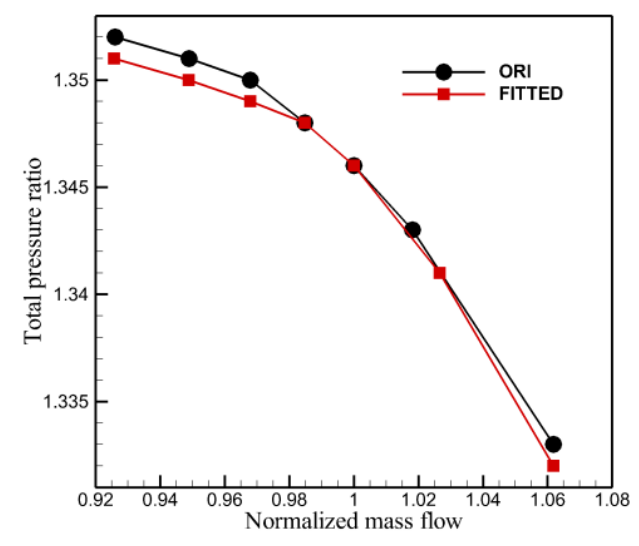

(a)

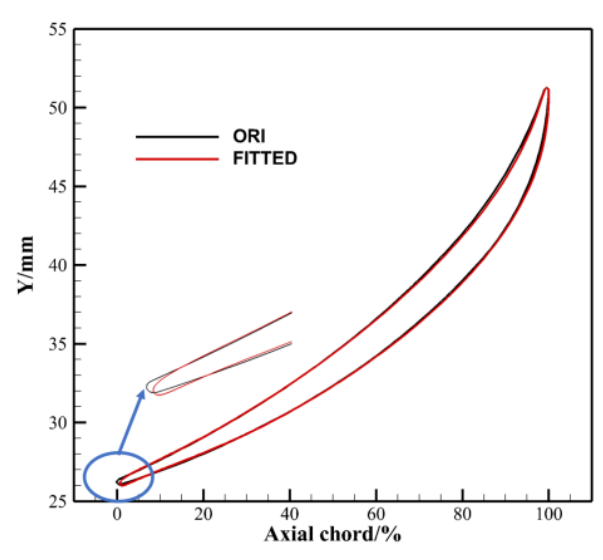

(b)

Figure 4. Comparison between prototype EAT and parameterized EAT

The tip of the blade is fitted with a Bézier curve, and six non-uniformly distributed control points are used in order to control the pressure surface profile, which controlls the shape of the pressure surface winglet. The method used for the generation of the sample space is Latin hypercube sampling. As samples are layered according to input probability, Latin hypercube sampling realises that samples with low probability are also represented by a value in the interval, and 
therefore a good balance can be achieved between sampling efficiency and running time. A total of 200 samples were generated, among which 11 samples had low grid quality and the effective sample size was 189. Figure 5 demonstrates the blade tip section parameterization process and the distribution of the control points.

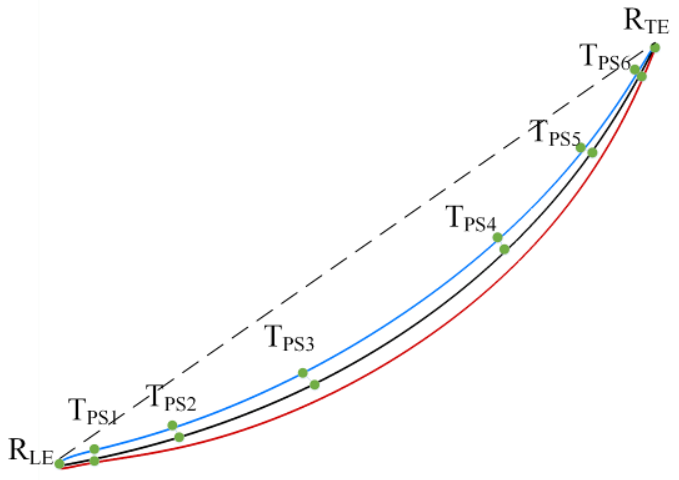

Figure 5. The distribution of the control points

\section{Optimization Design Frame}

Based on the above discussion, this paper adopted a parametric method based on Bézier curve fitting, and used six non-uniformly distributed pressure side control points in order to generate samples using the Latin hypercube experimental design method. The $\varepsilon-S V R$ model with a radial basis function (RBF) as the kernel was selected as the agent model, and a genetic algorithm (GA) was used as the optimization algorithm. The process of the optimization design frame is shown in the Figure 6.

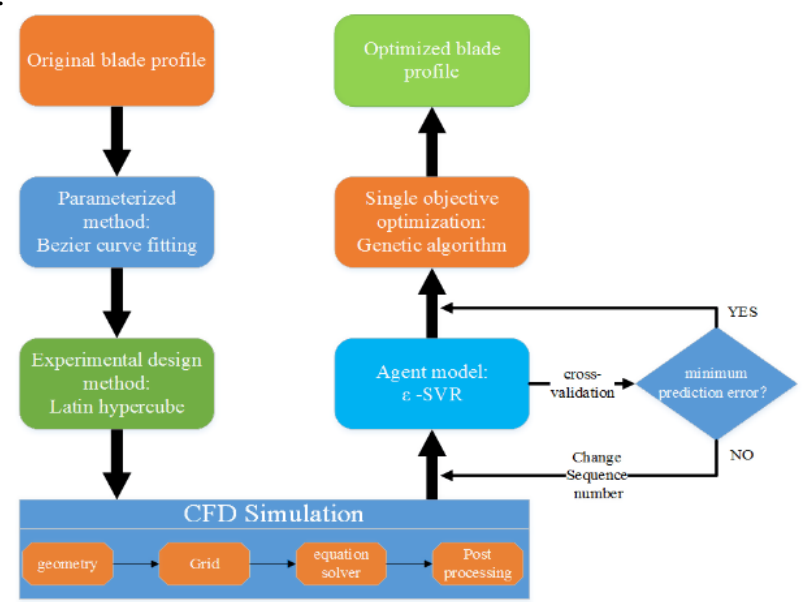

Figure 6. Optimization design frame

\section{OPTIMIZATION RESULT DISCUSSION}

For the EAT rotor which was studied in this paper, 200 samples were generated using Latin hypercube sampling, of which 189 were valid samples, and the grid quality of the remaining 11 invalid samples was too low. A CFD simulation was performed on the Intel i7-6700 3.40Ghz CPU, running in parallel using six threads of the processor. It took 661.5 hours. The sample space was used to train the $\varepsilon$-SVR model which was used, and a genetic algorithm was used in order to optimise the penalty parameter $\mathrm{C}$, the kernel parameter $\mathrm{g}$, and the value $\mathrm{p}$ of the loss function in $\varepsilon$-SVR. The prediction error after 9-fold cross-validation was $7.33 \%$. Based on the performance characteristics of pressure side winglets, the stability margin of the EAT rotor was taken as the objective function, the optimised blade tip winglet structure was obtained by genetic algorithm. The new optimised blade tip section was compared with the prototype as demonstrated in Figure 7. It can be observed that the optimised pressure side winglets obtained partial winglets that only covered part of the chord. The maximum thickness of the pressure surface was located at the position of $16 \%$ axial chord length, and the maximum thickness was 2 times that of the original maximum thickness. After the $75 \%$ chord length position, the optimised tip shape was consistent with the prototype. 


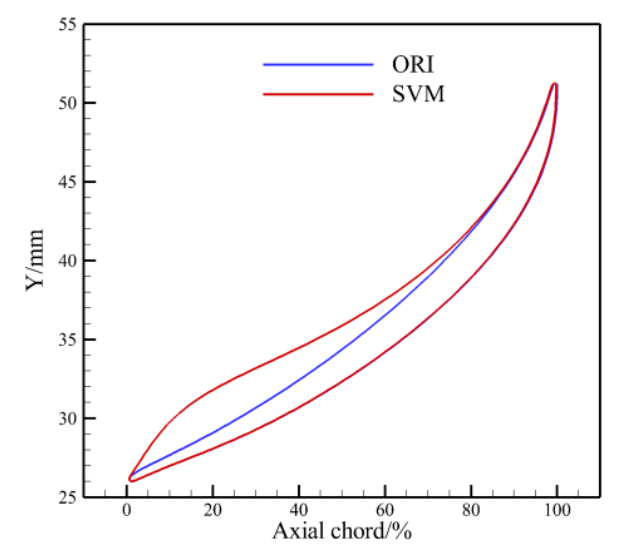

Figure 7. Comparison of the $\varepsilon$-SVR-GA optimized and original blade profile

As demonstrated in Figure 8, following optimisation, the multi-point flow of the rotor was calculated by CFD, and the characteristic lines of the optimisation blade were compared with the characteristic lines of the primary blade. Mass flow at the design point increased by $1.9 \%$ and the total pressure ratio decreased by $0.52 \%$. Mass flow at the near stall point increased by $0.91 \%$ and the total pressure ratio decreased by $0.88 \%$. After calculation, the stability margin of the prototype EAT rotor was $9.70 \%$, and the stability margin of EAT rotor optimised by the $\varepsilon$-SVR model was $12.16 \%$. This was an increase of $25.36 \%$ compared with the prototype and a difference of $4.1 \%$ from the stability margin predicted by the $\varepsilon$-SVR model.

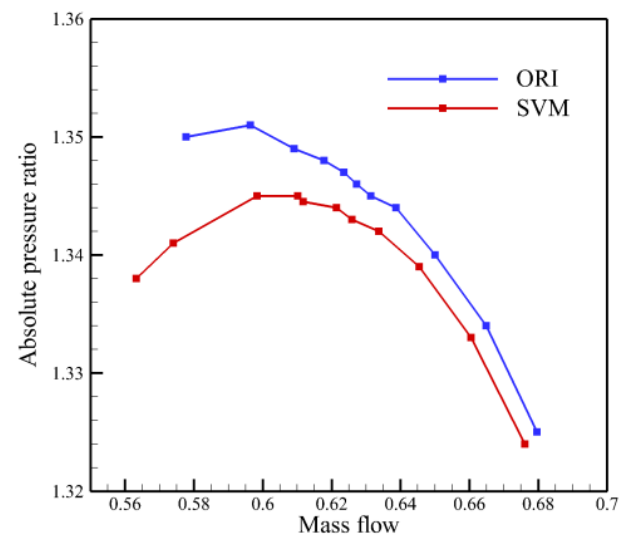

(a)

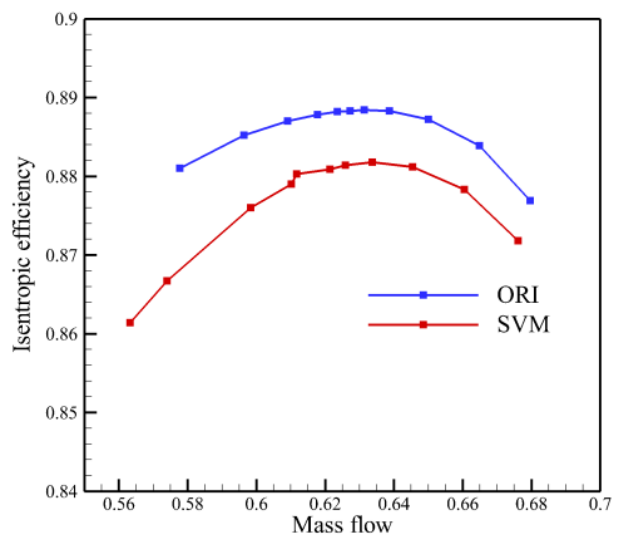

(b)

Figure 8. Comparison of the optimized and original blade on the total performance

The parameters used to quantificationally evaluate the aerodynamic performance of cascade are denoted as followed:

(1) flow coefficient

$$
\phi=\frac{V_{Z}}{U_{t}}
$$

(2) relative total pressure loss coefficient

$$
\Psi_{\text {LOSS }}=\frac{P_{R 1}^{*}-P_{R 2}^{*}}{0.5 \rho U_{t}^{2}}
$$

(3) relative static pressure coefficient

$$
C_{P S}=\frac{P_{S}-P_{a}}{0.5 \rho U_{t}^{2}}
$$

Figure 9 shows the distribution of circumferential average relative total pressure loss coefficient along with the prototype rotor and the optimised rotor outlet in the design condition. The flow coefficient of design point is 0.512. As can be seen from the Figure 9, the influence range of partial tip winglets on the distribution of relative total pressure coefficient of the rotor is $70 \%$ to $95 \%$ relative span, and it has no influence on the range of lower relative span area. Because the EAT prototype rotor was designed to achieve a high-load performance, the contraction of the meridional profile is sharp, and there is a strong radial flow inside the rotor, which made the high-energy fluid mixed with the fluid in lower relative span, so the relative total pressure loss coefficient below $70 \%$ relative span was negative. 


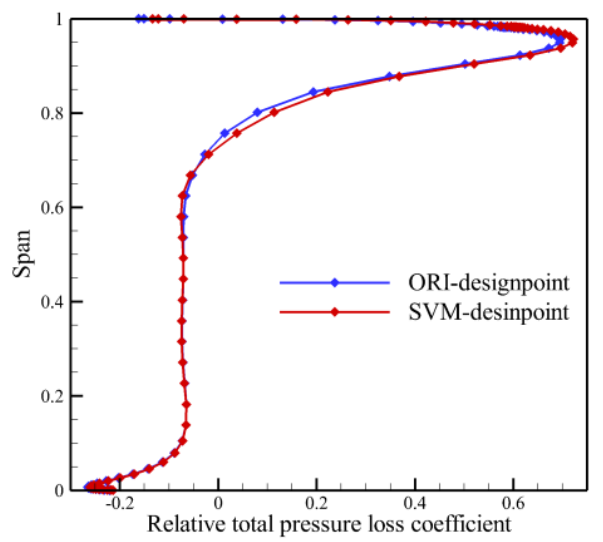

Figure 9. Spanwise distribution of circumferential average relative total pressure loss coefficient at the rotor outlet

Figure10 shows the S1 surface relative static pressure coefficient of $98 \%$ blade height in design condition. On the suction side of the blade, the tip leakage vortex created a region of low static pressure coefficient. The direction of the static chute is the same as that of the leakage vortex. Compared with the prototype rotor, the initial position of tip leakage vortex is closer to the trailing edge, and the angle between leakage vortex and the blade is reduced. The starting position closer to the trailing edge was conducive to the leakage vortex outflow channel, rather than the secondary leakage along with the circumferential flow to the pressure surface of the adjacent blades. The smaller angle with the blade can help the leakage vortex to absorb the low-speed fluid on the suction surface and delay the tendency of the suction surface boundary layer to separate under the action of the adverse pressure gradient.
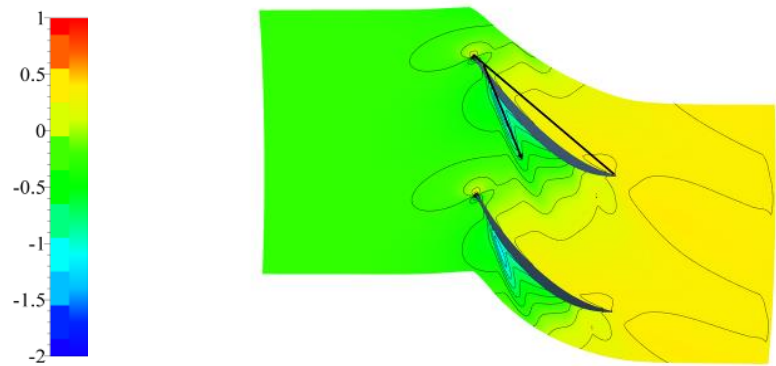

(a) Prototype

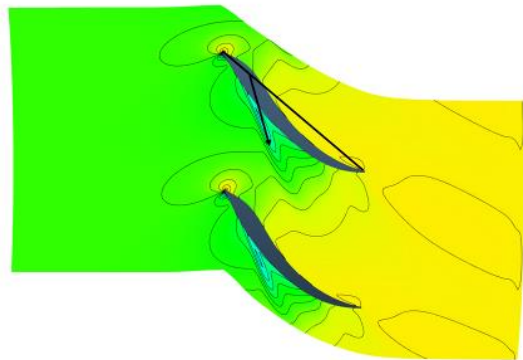

(b) $\varepsilon$-SVR-GA optimized

Figure 10. S1 surface relative static pressure coefficient of $98 \%$ blade height in design condition

In order to show the effect of partial winglets of the optimized rotor on rotor tip leakage flow more visually, Figure 11 displays the structure of the blade tip flow field of the prototype EAT rotor and the optimized rotor in design condition. The leakage flow streamlines through the blade tip pressure side of the rotor tip was obtained. The streamlines color is the relative velocity after using the blade tip tangent velocity dimensionless. In the design condition, due to the large aerodynamic load of the prototype rotor, part of the leakage fluid moved circumferential to the adjacent blades and crossed the pressure surface of the adjacent blades, forming a secondary leakage (DU 2010), the secondary leakage fluid was not involved in the main leakage vortex of next channel, but flowed out of the channel. Compared to the prototype rotor, under the blocking effect of partial winglets, the initial position of the leakage vortex is closer to the trailing edge, and no secondary leakage occurs, the leakage vortex is closer to the blade suction surface, which makes the interface between the leakage flow and the mainstream more distant from the pressure surface side of the adjacent blade. Partial winglets reduced the strength of tip leakage flow. 


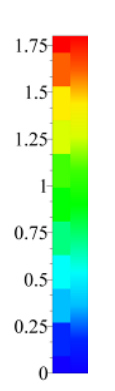

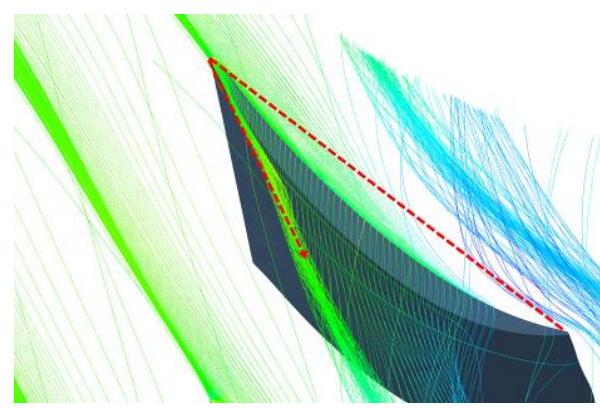

(a) Prototype

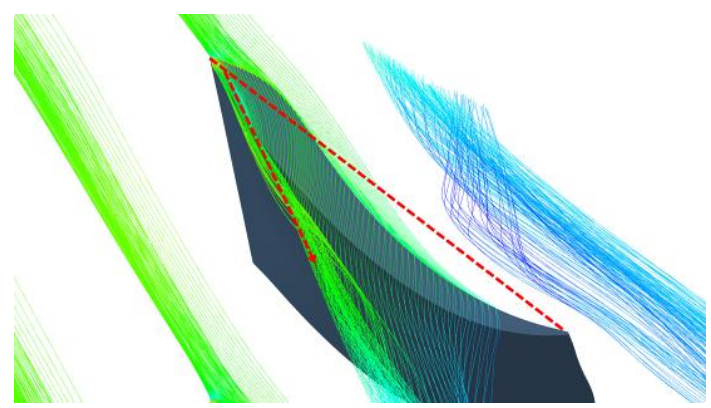

(b) $\varepsilon$-SVR-GA optimized

Figure 11. Flow field of the rotor blade tip area in design condition

Then the difference of flow between the prototype rotor and the optimized rotor in the near stall condition is analyzed. The flow coefficient of design point is 0.458 . Figure 12 displays the structure of the blade tip flow field of the prototype EAT rotor and the optimised rotor in near stall condition. Compared with the design condition, the angle between tip leakage vortex and suction surface is larger and the leakage flow was enhanced. Compared with the prototype rotor, the partial winglet of the optimized rotor can block the leakage fluid driven by pressure difference, and the position of the leakage vortex was closer to the blade suction surface, which can better entrain the low-energy fluid gathered on the suction surface. As the pressure surface thickness in the blade tip area increased, the leading edge point of the blade tip moved towards the pressure side, making the incidence angle relatively decreased, leading to the reduction of aerodynamic load, also the trend of separation. Secondary leakage occurred in both the prototype and the optimized rotor in the near-stall condition. A large number of secondary leakage fluids from the upper channel have appeared in the middle chord of the prototype blade tip, which has not been entrained by the main leakage vortex. Because the angle between the leakage vortex and suction side of the optimized rotor was small, some of the secondary leakage fluid flowed out of the flow path after being entrained by the main leakage vortex.

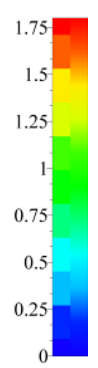

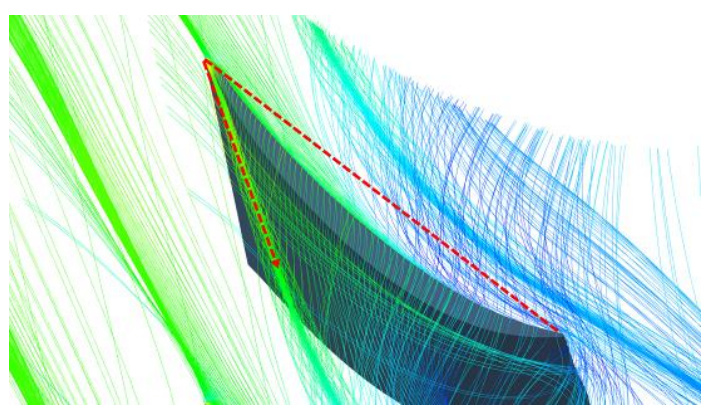

(a) Prototype

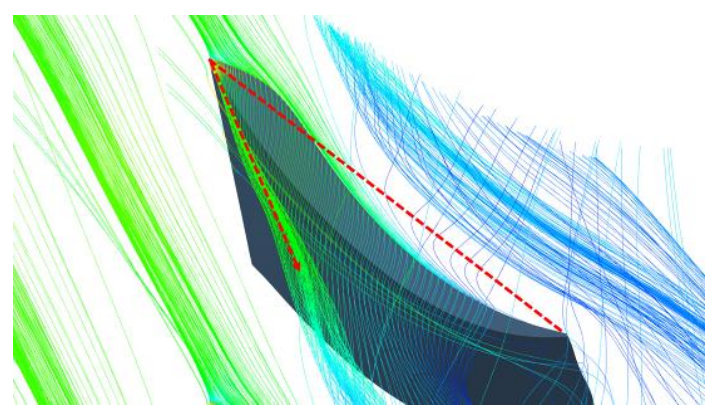

(b) $\varepsilon$-SVR-GA optimized

Figure 12. Flow field of the rotor blade tip area in near stall condition

\section{CONCLUSIONS}

In order to improve the stability margin of an electric auxiliary turbocharger (EAT), this paper generated a sample space with 189 valid samples by parameterizing the EAT rotor and using a Latin hypercube experimental design method, constructed the $\varepsilon$-SVR-GA optimization design platform, and verified it on the EAT rotor. Comparing it with the effect of a traditional BP-GA optimization system, and the different effect of full chord length winglets, following conclusions were reached:

1. As a result of statistical learning theory, the SVR model has better adaptability to small sample space. By choosing reasonable kernel functions and parameters, the size of the sample space can be reduced, the time required for optimization can be shortened, and computing resources can be saved. A genetic algorithm can be used in order to optimize the penalty parameter $\mathrm{C}$, kernel parameter $\mathrm{g}$ and the value $\mathrm{p}$ of the loss function in $\varepsilon$-SVR in a short time.

2. After the optimization design of the blade tip profile by using the optimization platform built in this paper, a partial winglet profile was obtained. Compared with the prototype EAT rotor, the stability margin increased by $25.36 \%$, the total pressure ratio at the design point decreased by $0.22 \%$, and the isentropic efficiency decreased by $0.74 \%$. The effectiveness of the optimized platform is verified.

3. Partial winglets can obstruct the flow of the leakage flow, reduce the angle between the main leakage vortex and the suction surface, improve the entrainment capacity of the main leakage vortex on the boundary layer of the casing endwall and the suction surface, increase the stability margin of the EAT rotor. For EAT rotor that needs to start and stop 
frequently, partial winglets can effectively improve its stability margin and reduce the additional mass of winglets, which is a more appropriate choice.

4. Due to the lack of similar public test data of "small diameter, medium hub ratio and high speed", it cannot be checked at present as the public test data of ROTOR37 used in related researches on transonic compressors. The validation work will carry out $r$ after the design process. According to the actual conditions, the test work will be divided into element blade profile plane cascade test, low-speed modelling test and full-size full-speed test.

\section{NOMENCLATURE}

$\begin{array}{llll}\pi & \text { absolute total pressure ratio } & \rho & \text { density } \\ m & \text { mass flow } & P_{\mathrm{s}} & \text { static pressure } \\ \eta & \text { isentropic efficiency } & P_{a} & \text { atmospheric pressure } \\ S M & \text { stability margin } & \omega & \text { blade load } \\ \phi & \text { flow coefficient } & \text { subscript } & \\ V_{Z} & \text { axial velocity } & \mathrm{PS} & \text { pressure side } \\ U_{t} & \text { tangent velocity of blade tip } & \mathrm{SS} & \text { suction side } \\ \Psi_{\text {Loss }} & \text { relative total pressure loss coefficient } & 1 & \text { inlet position } \\ P_{R}^{*} & \text { relative total pressure } & 2 & \text { outlet position }\end{array}$

\section{References}

HU C, et al, (2019). Research progress of electromechanical compound turbocharging technology. Vehicle Engine, 240(1), pp.1-6. doi:10.3969/j.issn.1001-2222.2019.01.001.

Arnold S,et al, (2001). Advanced turbocharging technologies for heavy-duty diesel engines. SAE Transactions, Vol. 110, Section 3, pp. 2048-2055. doi:10.4271/2001-01-3260

Katrasnik T., et al, (2003). Improvement of the dynamic characteristic of an automotive engine by a turbocharger assisted by an electric motor. Journal of Gas Turbines Power, 125(2), pp. 590-595. doi:10.1115/1.1563246

Biwersi S., et al. (2012). Electric compressor with high-speed brushless DC motor. MTZ worldwide, 73(12), pp. 50-53. doi:10.1007/s38313-012-0252-0

ZHAO Y S, et al, (2010). Technological development of vehicle electric assisted turbocharger. Vehicle \& Power Technology, 118(2), pp. 54-57. doi:10.16599/j.cnki.1009-4687.2010.02.010

LIU R L, et al, (2009). Status of application technology of electric supercharger. Small Internal Combustion Engine and Motorcycle, 38(2), pp. 41-43. doi:10.3969/j.issn.1671-0630.2009.02.010

SONG Y Y, et al, (2015). Comparison into the Effects of the Intake Manifold Length and Late Intake Valve Closure. Machinery Design \& Manufacture, 0(07), pp.69-71. doi:10.19356/j.cnki.1001-3997.2015.07.018

HAN S B, ZHONG J J (2012) Effects of Suction-Side Winglet on Aerodynamic Performance of Compressor Cascade at Different Incidences, Journal of Propulsion Technology 33(03), pp,384-390. doi:10.13675/j.cnki.tjjs.2012.03.016

Zhong J J, Han S B, (2017). Research progress of compressor tip leakage flow control using blade tip winglet. Journal of Propulsion Technology, 38(10), pp.45-52. doi:10.13675/j.cnki.tjjs.2017.10.005

SANG Z L, (2017). Experimental study on influence of blade tip geometries on the tip clearance flow of compressor cascade. M.S. Dalian Maritime University.

WU W Y, et al (2017), Effect of blade tip winglet on aerodynamic performance of high subsonic compressor cascade at different tip clearances. Journal of Dalian Maritime University, 43(02). pp,97-102. doi:10.16411/j.cnki.issn10067736.2017 .02 .015

WU W Y, (2018). Investigation of High Subsonic Compressor Cascade Tip Clearance Control Using Blade Tip Winglets. Ph.D. Dalian Maritime University.

Zhong J J, Han S B, (2016). Influence of blade tip winglet on the off-design performance of a transonic compressor rotor. Journal of Aerospace Power, 31(03).pp.647-658. doi:10.13224/j.cnki.jasp.2016.03.016

DU J, (2010). Investigation on the unsteady mechanism of tip leakage flow in transonic compressor/fan rotors. Ph.D. Chinese Academy of Sciences. 